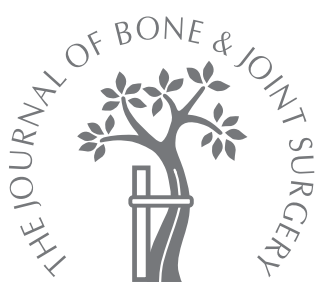

P. Harnett, R. Freeman, W. J. Harrison, L. C. Brown, V. Beckles

From Beit-Cure International Hospital, Blantyre, Malawi

P. Harnett, BSc, MB ChB, FRCS (Tr \& Orth), Specialist Registrar/Furlong Fellow W. J. Harrison, MA(Oxon), FRCS (Tr \& Orth), FCS (ECSA), Consultant Orthopaedic Surgeon, Medical Director Beit CURE International Hospital, PO Box 31236 Blantyre 3, Malawi.

In. Freeman, MA, FRCS (Tr \& Orth), Consultant Orthopaedic Surgeon

The Robert Jones and Agnes Hunt Orthopaedic and District Hospital NHS Trust, Oswestry, Shropshire SY10 7AG, UK.

= L. C. Brown, PhD, Medical Statistician

Department of Vascular

Surgery

Imperial College London, 4th Floor, Charing Cross Hospital, Fulham Palace Road, London W6 8RF, UK.

v. Beckles, BSc, MBBS, MRCS (Ed), Specialist Registrar Barking, Havering and Redbridge University Hospitals NHS Trust, Rom Valley Way, Romford, Essex RM7 OAG, UK.

Correspondence should be sent to Mr P. Harnett; e-mail: paulharnett2@yahoo.co.uk

(C2011 British Editorial Society of Bone and Joint Surgery doi:10.1302/0301-620X.93B3. $24450 \$ 2.00$

$J$ Bone Joint Surg [Br]

2011;93-B:404-8.

Received 2 June 2010; Accepted after revision 9 November 2010

\section{CHILDREN'S ORTHOPAEDICS}

\section{An accelerated Ponseti versus the standard Ponseti method}

\author{
A PROSPECTIVE RANDOMISED CONTROLLED TRIAL
}

\begin{abstract}
We conducted a prospective randomised controlled trial to compare the standard Ponseti plaster method with an accelerated method for the treatment of idiopathic congenital talipes equinovarus. The standard weekly plaster-change method was accelerated to three times per week. We hypothesised that both methods would be equally effective in achieving correction. A total of 40 consecutive patients (61 feet) were entered into the trial. The initial median Pirani score was 5.5 (95\% confidence interval 4.5 to 6.0 ) in the accelerated group and 5.0 (95\% confidence interval 4.0 to 5.0$)$ in the standard control group. The scores decreased by an average 4.5 in the accelerated group and 4.0 in the control group. There was no significant difference in the final Pirani score between the two groups (chi-squared test, $p=0.308$ ). The median number of treatment days in plaster was 16 in the accelerated group and 42 in the control group $(p<0.001)$. Of the 19 patients in the accelerated group, three required plaster treatment for more than $\mathbf{2 1}$ days and were then assigned to the standard control method. Of the $\mathbf{4 0}$ patients, $\mathbf{3 6}$ were followed for a minimum of six months.

These results suggest that comparable outcomes can be achieved with an accelerated Ponseti method. The ability to complete all necessary manipulations within a three-week period facilitates treatment where patients have to travel long distances.
\end{abstract}

Congenital talipes equinovarus (CTEV) occurs in 1.2 per 1000 live births in Europe, but in some developing countries the incidence is double. ${ }^{1}$ In approximately $20 \%$ of patients, CTEV is associated with other congenital abnormalities. $^{2,3}$ A variety of classification systems based on clinical examination have been used, the most widely used being that of Pirani, ${ }^{4}$ which has been shown to have good interobserver reliability and reproducibility. ${ }^{2,5}$ The Ponseti method has transformed the management of children with CTEV producing good long-term results $^{6-8}$ and in the last two decades has gained wide acceptance in the worldwide orthopaedic community. ${ }^{9}$ The standard Ponseti method uses weekly foot and leg plaster changes to gradually correct the deformity, using a strictly defined sequence of moulded plaster changes. The final deformity to be corrected is equinus, which often requires a percutaneous tendo Achillis tenotomy followed by a final plaster for three weeks. Once plastering is finished, children are placed in a foot-abduction brace. Percutaneous tendo Achillis tenotomy and later tibialis anterior transfer may be used but are not always necessary. ${ }^{10}$ The Ponseti technique is well established and has been shown to be highly effective, so why seek to alter it? The Malawi population often have to travel long distances for treatment, and this may take days for some patients. Transport is expensive and two months away from home, seven plaster changes and then three weeks in plaster following tenotomy, can cause considerable disruption to family life. In addition, keeping a plaster clean and dry for a week can be challenging and failure to do so may result in a loss of position. We hypothesised that a two-day interval might be as effective as weekly plaster changes. In order to avoid plaster changes over the weekend, we proposed to change the plasters three times a week (on Monday, Wednesday and Friday). The accelerated method would suit in-patient or outpatient treatment, and the final plaster could be removed locally. This could reduce hospital stay to three weeks.

\section{Patients and Methods}

A protocol for a prospective randomised controlled trial was drawn up to compare an accelerated Ponseti method with the standard Ponseti method. Ethical approval was obtained from the Malawi College of Medicine Research and Ethics Committee. Power calculations were performed using a Pirani score of $>1.0$ as significant. The study aimed to recruit 40 patients. 
Table I. Comparison of characteristics between randomised groups

\begin{tabular}{|c|c|c|c|}
\hline Variable $^{*}$ & $\begin{array}{l}\text { Accelerated group } \\
(n=19)\end{array}$ & $\begin{array}{l}\text { Control group } \\
(n=21)\end{array}$ & $\begin{array}{l}\text { p-value from comparative } \\
\text { test }^{\dagger}\end{array}$ \\
\hline Median age in days (IOR) & 21 (7 to 48$)$ & $29 \quad(10$ to 55$)$ & 0.32 \\
\hline Number of males $(\%)$ & $10 \quad(53)$ & $10 \quad(48)$ & 0.75 \\
\hline Number with both feet treated (\%) & $9 \quad(47)$ & $10 \quad(48)$ & 0.99 \\
\hline Tenotomy required (\%) & 15 (79) & $11 \quad(52)$ & 0.079 \\
\hline Median baseline Pirani score & $5.5(4.5$ to 6.0$)$ & $5.0(4.0$ to 6.0$)$ & 0.143 \\
\hline Median Pirani score at tenotomy or end of treatment & $0.5(0.5$ to 1.0$)$ & $0.5(0$ to 1.0$)$ & 0.299 \\
\hline Median Pirani score at 6 months' follow-up & $0 \quad(0$ to 1.0$)$ & $0.5(0$ to 0.5$)$ & 0.630 \\
\hline
\end{tabular}

* continuous variables presented as median (IOR, interquartile range) as data were skewed

† Mann-Whitney $U$ test for continuous variables, chi-squared test for categorical variables

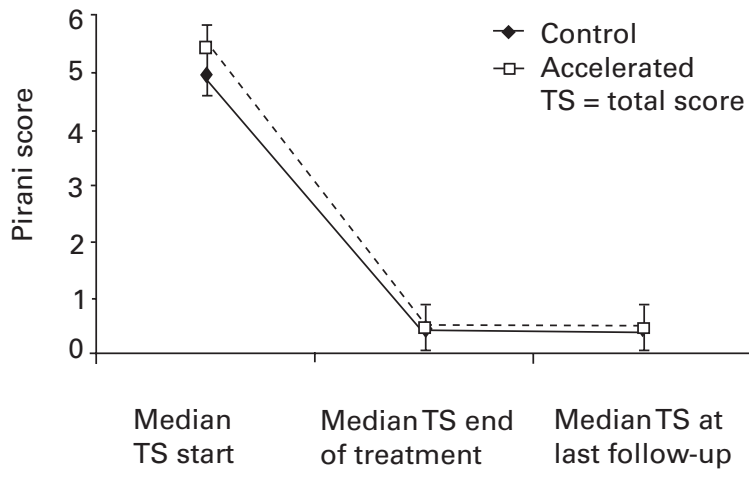

Fig. 1

Graph showing median change in Pirani scores.

Inclusion criteria were a diagnosis of idiopathic CTEV, age $<90$ days, local residency, and informed consent from the patient's guardian or parents.

The patients were randomised by computer-generated numbers to either the standard Ponseti method or the accelerated method. Randomisation occurred at the patient level, with both feet being treated using the same method. All feet were scored using the Pirani method, ${ }^{4}$ recorded by an independent assessor at each visit. Plaster treatment was continued until clinical correction was achieved. A percutaneous tendo Achillis tenotomy was performed if dorsiflexion was $<10^{\circ}$ at the end of manipulation and plastering. Both groups were put into plaster, following tenotomy, for three weeks. The defined endpoint of treatment for both groups, labelled 'treatment time in plaster' refers to the number of days in plaster prior to a tenotomy. Within the accelerated group we defined failure as a Pirani score of $>1.0$ at 21 days of plaster treatment. Both groups were given abduction braces to wear for three years following their plaster treatment, 23 hours per day for the first three months, followed by night-time bracing until the child's third birthday, in accordance with the standard local Ponseti programme. All patients in the study lived locally, but to minimise the loss to follow-up, transportation costs were refunded. All patients were treated as outpatients, thereby reducing any bias from altered compliance and enabling us to directly compare the efficacy of the two methods in terms of correction of the deformity. Follow-up was planned at monthly intervals for the first year and at sixmonthly intervals up to the age of three years.

Statistical analysis. Data analysis was carried out using STATA version 10.0 (Stata Corp., College Station, Texas). In order to compare randomised groups for categorical data we used chi-squared tests and Mann-Whitney U tests for continuous variables. A p-value $<0.05$ was considered statistically significant.

\section{Results}

A total of 40 children (61 feet) with idiopathic CTEV were recruited into the trial, 21 of whom were bilateral. The mean age of the children was 31 days ( 7 to 55$)$; the data were positively skewed with a median interquartile age of 18 days ( 7 to 52 ). A total of 19 patients (29 feet) were allocated to the accelerated protocol and 21 to the standard control treatment (32 feet).

There were no missing data at baseline and characteristics were compared between the two groups (Table I). There were no significant differences between the groups before treatment and no significant difference in the final Pirani score (Fig. 1) (chi-squared test, $\mathrm{p}=0.308$ ). A consort diagram describing the flow of patients through the trial can be seen in Figure 2. In the accelerated group three of the 19 patients did not respond to the treatment and crossed over to the standard Ponseti method. One of the 'crossover' patients required a tibialis anterior transfer to achieve correction, whereas the other two achieved correction with weekly manipulation and plaster changes. One child from the control group died of malaria while still in plaster and the extent of correction could not be confirmed, and this patient was censored as non-corrected at death. Of the 40 patients, three $(7 \%)$ were 'not corrected' (Pirani score $>1.0$ ), one from the accelerated group and two from the control group and they required a tibialis anterior transfer to achieve correction. There was no difference between the number of plaster changes between the groups: median five 


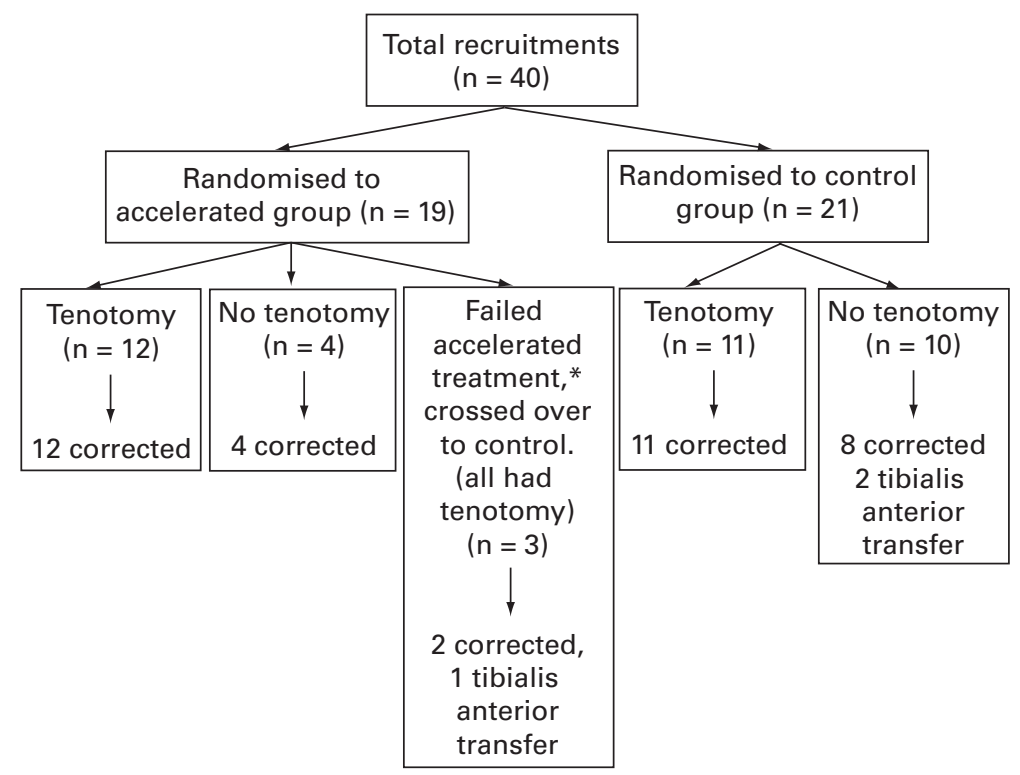

Fig. 2

CONSORT diagram demonstrating the flow of patients through the trial (* failed refers to a Pirani score $>1.0$ at 21 days of plaster treatment).

\begin{tabular}{|c|c|c|c|c|}
\hline Outcome ${ }^{*}$ & $\begin{array}{l}\text { Accelerated group } \\
(n=19)\end{array}$ & $\begin{array}{l}\text { Control group } \\
(n=21)\end{array}$ & $\begin{array}{l}\text { Crude Cox's hazard ratio } \\
\left(95 \% \mathrm{Cl}^{+}\right)(p \text {-value) }\end{array}$ & $\begin{array}{l}\text { Adjusted }{ }^{\ddagger} \text { Cox's hazard ratio } \\
(95 \% \mathrm{Cl}) \text { (p-value) }\end{array}$ \\
\hline $\begin{array}{l}\text { Change in Pirani score between baseline } \\
\text { and end of treatment }\end{array}$ & $-4.5(-3.5$ to -5.0$)$ & $-4.0(-3.5$ to -5.0$)$ & $N / A^{\S}$ & $\mathrm{N} / \mathrm{A}$ \\
\hline Treatment time in plaster & $16 \quad(14$ to 20$)$ & $42 \quad(35$ to 84$)$ & $3.32(1.55$ to 7.11$)(0.002)$ & $7.26(2.43$ to 21.7$)(<0.001)$ \\
\hline
\end{tabular}

(four to eight) for the accelerated group and five (four to seven) for the control group.

All patients were analysed within their randomised group according to the intention-to-treat principle. Table II presents the results of Cox regression for time to correction by group.

The crude hazard ratios from the Cox regression model, indicate that on average, at any given time during the study, the accelerated group were 3.3 times more likely to achieve correction than the control group. After adjustment for the slight differences between groups at baseline, mainly lower Pirani scores in the control group and a higher number of tenotomies in the accelerated group, the adjusted hazard ratio suggests that, on average, the accelerated group were 7.3 times more likely to achieve correction than the control group with regard to time to correction.

For both crude and adjusted models, these hazard ratios are strongly statistically significant, and this difference is demonstrated clearly by the Kaplan-Meier curves for time to correction (Fig. 3).

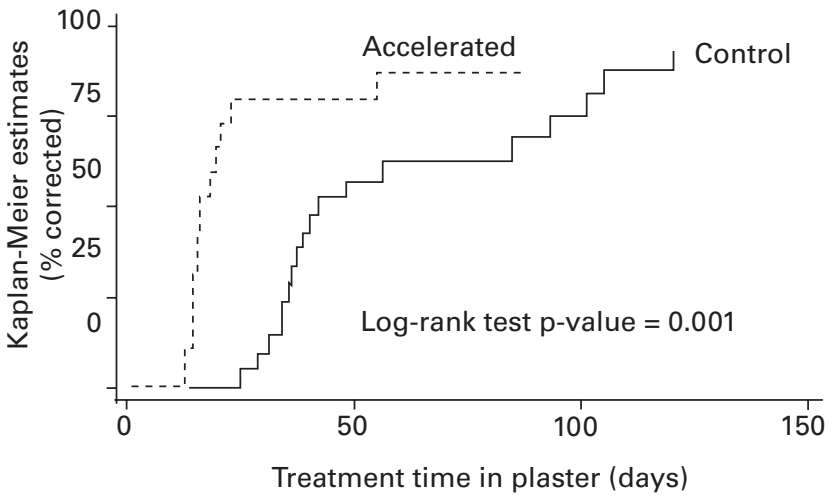

Fig. 3

Graph showing Kaplan-Meier estimates for time to correction by randomised group, with p-values for log-rank tests.

Follow-up and recurrence rates. Of the 40 patients, 36 (55 feet) were followed up for at least six months. Follow-up in the accelerated group was for a mean of 258 days (70 to 348 ) and in the control group for a mean of 244 days ( 25 to 


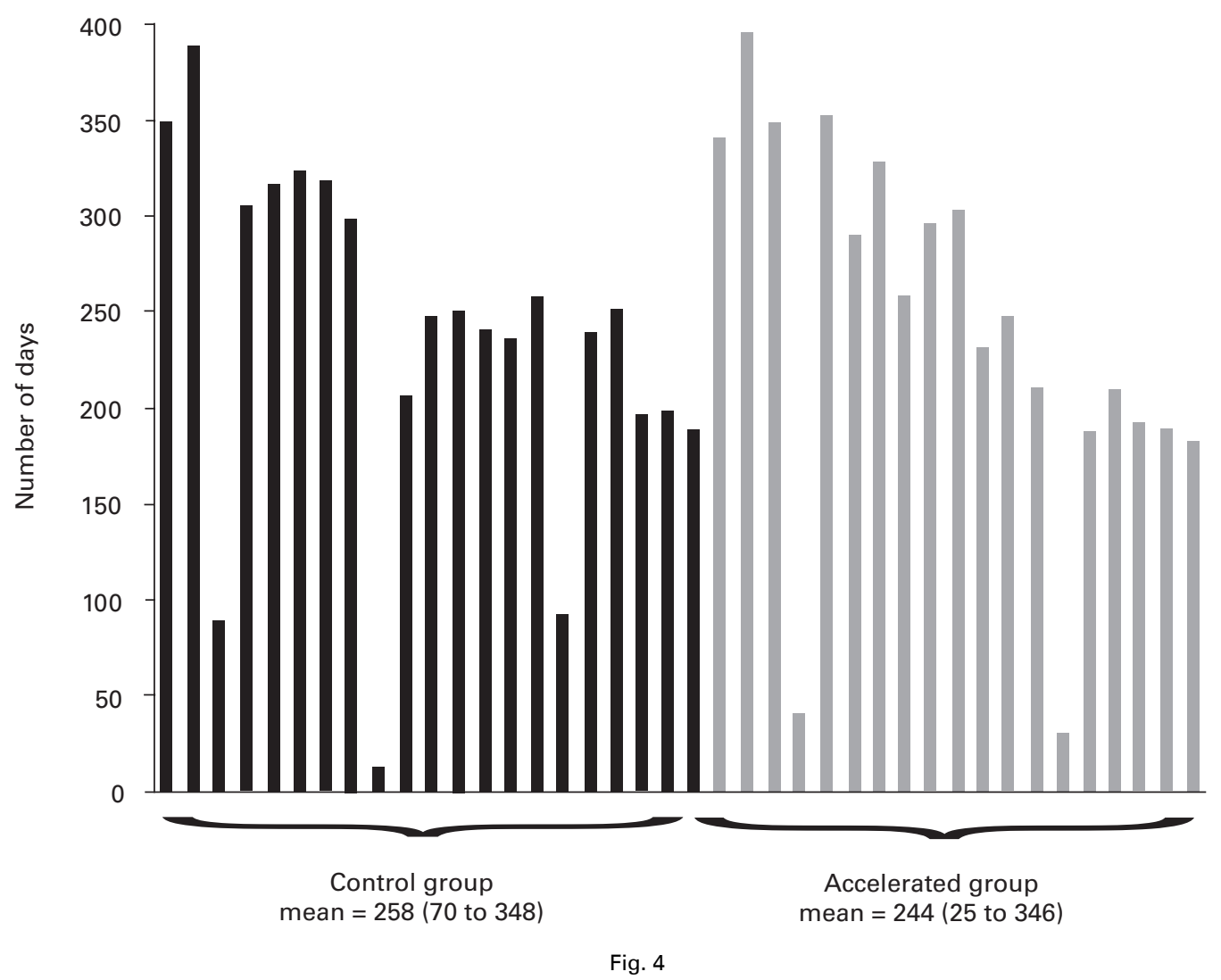

Bar chart showing the number of days of follow-up in the 40 patients.

346) (Fig. 4). There were no episodes of recurrence at six months (Table I). One control patient died during treatment, the other four were lost to follow-up after plaster treatment, two from the control group and two from the accelerated group.

\section{Discussion}

The Ponseti method is widely accepted and practised, giving reliably good long-term results. Our results suggest that in the short term comparable results can be achieved with an accelerated method, changing the plaster three times per week. Morcuende et al $^{11}$ undertook a non-randomised retrospective study of 230 patients (319 clubfeet) over an 11year period. Patients were assigned to a five- or seven-day frequency of plaster changes based solely on geography. The study population was heterogenous with regard to age and previous treatment; $67 \%$ were less than four months of age, $16 \%$ were between four and six months and $16 \%$ were more than six months of age; $72 \%$ had some form of treatment before their initial treatment in their unit. The severity was determined by the number of plaster changes needed to obtain correction. The authors found that an accelerated five-day interval can be equally effective. The average number of plasters (four) did not differ between groups $(\mathrm{p}=0.85)$. Percutaneous tendo Achillis tenotomy was performed in $85 \%$ of the five-day group and $81 \%$ in the seven-day group $(\mathrm{p}=0.4)$. The recurrence rates were $8 \%$ and $7 \%$ for the five- and seven-day groups, respectively, with an 8.5 times greater risk of recurrence if compliance with abduction bracing was not adhered to $(<10$ hours per day) ( $\mathrm{p}=0.0001)$. It was thought that further acceleration might give rise to problems with swelling in the plaster, but no child in our study developed this problem.

The tenotomy rate in the accelerated group was $79 \%$, compared to $52 \%$ in the control group ( $\mathrm{p}=0.07$ ), which was not statistically significant. A number of authors have described a percutaneous tendo Achillis tenotomy rate for the correction of persistent equinus using the Ponseti method of $70 \%$ to $80 \%$. ${ }^{1,8,9}$

In our study the accelerated group had a slightly more severe deformity as defined by the initial Pirani score, accelerated, 5.5 (4.5 to 6.0), control, 5.0 (4.0 to 6.0); although not statistically significant $(\mathrm{p}=0.143)$, this may account for the greater requirement for a tenotomy in the accelerated group. This characteristic was identified by Scher et al, ${ }^{12}$ who related higher Pirani scores to the need for a tenotomy. In the accelerated group three of 19 patients required plaster treatment for more than 21 days, and were then 
assigned to the standard method. We were unable to discover any characteristics that might predict which patients were unsuitable for the accelerated method.

A tibialis anterior transfer was required in three of the 40 patients $(7 \%)$ to achieve correction. We perform this procedure in younger patients (mean of six months) than recommended using the standard Ponseti protocol, as longer follow-up for two to three years is difficult in our population.

If the longer term results of the accelerated method continue to be comparable to those of the standard Ponseti method, it can offer patients a number of benefits. The cost to the parents can be reduced by admitting the patient for three weeks or arranging local accommodation in a hostel. This will involve less time off work and less time away from other dependents. In-patient treatment should improve compliance during the plaster programme. In this study we improved compliance by funding transport. If tenotomy is required it may be possible to have the final plaster removed and the boots and bars fitted in a clinic closer to home.

Osteopenia after immobilisation in above-knee plasters has been reported in neglected clubfeet (mean age 3.9 years) by Lourenco and Morcuende, ${ }^{13}$ but these findings resolved within a few months after plaster removal. It is possible that the accelerated method might reduce this problem still further.

Pirani, Zeznik and Hodges ${ }^{14}$ have described changes in the hindfoot cartilage anlage on MRI scans following treatment by the standard Ponseti method. It would be interesting to compare the changes seen on an MRI scan in an accelerated group with those in the control group.

In the Western world there is a drive to improve patient choice, and if the accelerated method is equally effective, this could provide parents with the alternative of more rapid treatment. Other potential advantages are a reduction in the likelihood of plasters slipping and the chance for more intensive education regarding the importance of boots and bars, with more visits over a shorter period.

The incidence of clubfoot in Malawi is 3 per 1000 live births, which is double that of most Western countries. ${ }^{1}$ Most developing countries have the same problems of high transport cost but a low admission cost. Our findings could have a major effect on the treatment of clubfoot in these countries.

The strength of this study is that it was a prospective randomised controlled trial comparing the accelerated method with the standard treatment. Unfortunately, follow-up is very short, less than a year in all but two patients. We will need to follow recurrence rates closely in order to investigate whether any patients treated by the accelerated method has late recurrence. The most significant factor with regard to recurrence during follow-up is compliance with the abduction brace, ${ }^{11}$ which we did not analyse. Haft,
Walker and Crawford ${ }^{15}$ reported a five times greater chance of recurrence in patients whose parents were noncompliant with bracing. So far there is no evidence to suggest that compliance with bracing differs between the two groups in this study. However, our experience with compliance among the rural community living in Malawi is poor.

In conclusion, the initial results suggest that an accelerated Ponseti method with plaster changes three times a week appears to be as effective as the standard Ponseti method. We hope that this will provide a useful alternative, particularly for patients who have to travel long distances for treatment and for those who find it difficult to comply with weekly plaster treatment.

\section{Listen live \\ Listen to the abstract of this article at www.jbjs.org.uk/interactive/audio}

We thank the rehabilitation therapy team of K. Khoya, J. Kayira, and the Malaw Club Foot Co-ordinator H. Salaka and Dr K. Phiri, Epidemiologist, Malawi-Liverpool Wellcome Trust, for their contributions. Acknowledged Fellowship Funding via: Furlong Research Charitable Foundation.

No benefits in any form have been received or will be received from a commercial party related directly or indirectly to the subject of this article.

\section{References}

1. Mkandawire NC, Kaunda E. Incidence and patterns of congenital talipes equinovarus (clubfoot) deformity at Queen Elizabeth Central Hospital, Banter, Malawi. East Afr J Surg 2004;9:28-31

2. Siapkara A, Duncan R. Congenital talipes equinovarus: a review of current management. J Bone Joint Surg [Br] 2007;89-B:995-1000.

3. Chung CS, Nemechek RW, Larsen IJ, Ching GH. Genetic and epidemiological studies of clubfoot in Hawaii: general and medical considerations. Hum Hered 1969;19:321-42.

4. Pirani S. Pirani severity scoring. In: Staheli I, ed. Clubfoot: Ponseti management Third ed. Global-HELP, 2009:27. http://www.global-help.org/Publications/books/ (date last accessed 22 November 2010).

5. Flynn JM, Donohue M, Mackenzie WG. An independent assessment of two clubfoot-classification systems. J Pediatr Orthop 1998;18:323-7.

6. Cooper DM, Dietz FR. Treatment of idiopathic clubfoot: a thirty-year follow-up note. J Bone Joint Surg [Am] 1995;77-A:1477-89.

7. Morcuende JA, Dolan LA, Dietz FR, Ponseti IV. Radical reduction in the rate of extensive corrective surgery for clubfoot using the Ponseti method. Pediatrics 2004;113:376-80

8. Ponseti IV. Congenital clubfoot: fundamentals of treatment. Oxford, Oxford University Press, 1996.

9. MacNicol M. The management of club foot: issues for debate. J Bone Joint Surg [Br] 2003;85-B:167-70.

10. Laaveg SJ, Ponseti IV. Long-term results of treatment of congenital club foot. $J$ Bone Joint Surg [Am] 1980;62-A:23-31.

11. Morcuende J, Abbasi D, Dolan L, Ponseti I. Results of an accelerated Ponseti protocol for clubfoot. J Pediatr Orthop 2005;25:623-6.

12. Scher DM, Feldman DS, Van Bosse HJ, Sala DA, Lehman WB. Predicting the need for tenotomy in the Ponseti method for correction of clubfeet. J Pediatr Orthop 2004;24:349-52.

13. Lourenco AF, Morcuende JA. Correction of neglected idiopathic club foot by the Ponseti method. J Bone Joint Surg [Br] 2007;89-B:378-81.

14. Pirani S, Zeznik L, Hodges D. Magnetic resonance imaging study of the congenital clubfoot treated with the Ponseti method. J Pediatr Orthop 2001;21:719-26.

15. Haft GA, Walker CG, Crawford HA. Early clubfoot recurrence after use of the Ponseti method in a New Zealand population. J Bone Joint Surg [Am] 2007;89-A:487-93. 